\title{
A new universal formula for atoms, planets, and galaxies
}

\author{
Tony Barrera, Bo Thelin \\ Barrera Kristiansen AB Granitvägen 12B, S-752 43 Uppsala, Sweden \\ Solarphotonics HB Granitvägen 12B, S-752 43 Uppsala, Sweden
}

\begin{abstract}
In this paper a new universal formula about the rotation velocity distribution of atoms, planets, and galaxies is presented. It is based on a new general formula based on the relativistic Schwarzschild/Minkowski metric, where it has been possible to obtain expressions for the rotation velocity - and mass distribution versus the distance to the atomic nucleus, planet system centre, and galactic centre. A mathematical proof of this new formula is also given. This formula is divided into a Keplerian(general relativity)-and a relativistic(special relativity) part. For the atomic-and planet systems the Keplerian distribution is followed, which is also in accordance with observations.

According to the rotation velocity distribution of the galaxies the rotation velocity increases very rapidly from the centre and reaches a plateau which is constant out to a great distance from the centre. This is in accordance with observations and is also in accordance with the main structure of rotation velocity versus distance from different galaxy measurements.

Computer simulations were also performed to establish and verify the rotation velocity distributions in the atomic - planetary- and galaxy system, according to this paper. These computer simulations are in accordance with observations in two and three dimensions. It was also possible to study the matching percentage in these calculations showing a much higher matching percentage between theoretical and observational values by this new formula.
\end{abstract}

Keywords: Atomic Physics, Astrophysics, Planetary physics, Spiral galaxies, Theory of Relativity, and Universal Formula

\section{Introduction}

It is common in physics that electron circular movement around atoms and planetary circular movement around the sun follow the usual Keplerian relationship $\mathrm{V} \alpha(1 / \sqrt{ } \mathrm{R})$, where $\mathrm{V}$ is the rotation speed and $\mathrm{R}$ is the distance to the nucleus of the atom or the centre of the sun respectively.

The mass of a spiral galaxy can be determined from the dependence of its rotational velocity as a function of the distance from the centre of the galaxy. Such a rotational curve has been determined from gas and stars in the distant parts of our galaxy, far beyond our distance to the centre. Unexpectedly, it does not follow the Keplerian decrease in which the circular rotation velocity $\mathrm{V}$ decreases $\alpha \mathrm{R}^{-1 / 2}$ where $\mathrm{R}$ is the distance to the centre. According to the 3:rd law of Kepler the mass of a galaxy can be expressed as :

$$
\mathbf{M}=\mathbf{V}^{2} \mathbf{R} / \mathbf{G}
$$

and the rotation velocity as:

$$
\mathbf{V}=(\mathbf{G} \mathbf{M} / \mathbf{R})^{1 / 2}
$$

where $\mathrm{G}$ is the gravitation constant.

By using these formulas it is possible to determine the mass and rotation speed at a certain distance of the galaxy. According to these equations both mass and rotation velocity will

decrease with increasing distance, which is established today.

In the 1970s and 1980s radio astronomers discovered that the spiral rotation velocity

remains constant with increasing radius Freeman ( 1 ), Rubin and Ford ( 2 ). They studied neutral hydrogen clouds at $21-\mathrm{cm}$ radio wavelength and in the optical wavelength in spiral galaxies and found non-Keplerian rotation curves. These facts were not in accordance with the established views and came as a shock to the establishment.

This is illustrated in Combes et al.( 3 ) (Figs 3.1-3.3), where the velocities of many spiral galaxies increase the velocity very rapidly at small distances up to a constant plateau at larger distances from the galaxy centre. Astronomers discovered that many galaxies rotated at very high velocities.

To explain this most astronomers believe that this is caused by introducing dark matter into the Keplarian equations above and by keeping the galaxies together. They believe that most matter in a galaxy consists of dark matter and only a minor part consists of ordinary matter which emits light.

In Barrera and Thelin ( 4 ) we have presented a new formula about the formation of galaxies. It is based on the relativistic Schwarzschild/ Minkowski metric, Schwarzschild ( 5 ), Einstein ( 6 ), where it has been possible to obtain a formula for the rotation velocity and also a density distribution versus distance to the galactic centre. Similar rotation velocity profiles to our new formula have also been observed from data published in established 
books in this field. These profiles are in accordance with observations, as is seen in equations ( 1-10) in Barrera and Thelin ( 4 ). Computer simulations of equations 19 and 22 of Barrera and Thelin ( 4 ) were also performed to establish and verify the velocity and density distributions suggested in that paper.

According to this rotation velocity formula, the rotation velocity increases very rapidly from the centre and reaches a plateau which is constant out to a great distance from the centre. This has also been observed in many papers, Sofue and Rubin.( 7 ) and Combes et al.( 3 ).

In this paper an improvement of the rotation formula for galaxies equation(19) of

Barrera and Thelin ( 4 ) is made and is seen in equation ( 33 ) in this paper. The approximate formula of Barrera and Thelin ( 4 ) is also seen in equation 15 of this paper. The new improved rotation formula in equation 33 is divided into one Keplerian (general relativity) part and one relativistic (special relativity) part, which also makes it possible to use this formula for atoms, planets, and galaxies. A mathematical proof of this new universal formula is also given. Computer simulations in 2 and 3 dimensions by this new formula are also achieved, giving it a strong support of the appearance of atoms, planets, and galaxies. In Table 1 galaxy parameters and matching between theoretical and observational data are also studied for a number of galaxies.

\section{Keplarian relationships of atom - and planetary physics}

According to Gasiorowicz 8 ) p.16 the relation between Coulombs law and the circular centripetal force of an electron around an atom is

$\left(\mathrm{Ze}^{2} / 4 \pi \epsilon_{0} \mathrm{r}^{2}\right)=\left(\mathrm{m}_{\mathrm{e}} \mathbf{v}^{2} / \mathbf{r}\right)$

where $\mathrm{m}_{\mathrm{e}}$ is the electron mass and $\mathrm{e}$ is the electron charge and $\mathrm{v}$ is the electron speed and $\mathrm{r}$ is the distance to the nucleus which gives

$v=\left(Z^{2} / 4 \pi \epsilon_{0} m_{e} r\right)^{1 / 2}$

which follows the Keplerian relationship $\mathrm{v} \alpha\left(1 / \mathrm{r}^{1 / 2}\right)$ between rotation velocity and distance.

When using the angular momentum in the Bohr postulate according to Gasiorowicz ( 8 ) p.16

$\mathbf{m}_{\mathrm{e}} \mathbf{v} \mathbf{r}=\mathbf{n}(\mathbf{h} / 2 \pi)$

where $\mathrm{n}$ is the principal quantum number, the following expression of $\mathrm{v}$ is obtained

$v=\left(Z \mathrm{e}^{2} / 2 \mathbf{\epsilon}_{0}\right)(\mathbf{1} / \mathrm{h} \mathrm{n}) \alpha(1 / \mathrm{n})$

where $v \alpha(1 / \mathrm{n})$.

These proportionalities between rotation velocity versus distance and principal quantum number will be shown in section 5 by computer simulations in 2 and 3 dimensions.

Planets follow a similar equation to equation 2 following the 3:rd law of Kepler. The exact

formula between the planet rotation speed $\mathrm{V}$ and the masses $\mathrm{m}$ for the planet and $\mathrm{M}$ for the sun is

$$
\mathbf{V}=(\mathbf{G}(\mathbf{m}+\mathbf{M}) / \mathbf{r})^{1 / 2}
$$

for circular motion according to Lang ( 9 ) p.542.

\section{Galaxies. The use of Schwarzschild metric}

According to Lang ( 10 ) p.146 a spherically symmetric gravitational field outside a massive nonrotating body in vacuum, can follow the Schwarzschild expression, where the line element ds becomes :

$\mathrm{ds}^{2}=\left(1-2 \mathrm{GM} / \mathrm{c}^{2} \mathbf{r}\right) \mathrm{c}^{2} \mathrm{dt}^{2}-\left(1-2 \mathrm{GM} / \mathrm{c}^{2} \mathbf{r}\right)^{-1} \mathrm{dr}^{2}-\mathrm{r}^{2} \mathrm{~d} \theta^{2}-\mathrm{r}^{2} \sin ^{2} \theta$

d $\psi^{2}$

Here $\mathrm{r}, \theta$ and $\psi$ are spherical coordinates whose origin is at the centre of the massive object

with the mass $\mathrm{M}, \mathrm{G}$ is here the gravitational constant and $\mathrm{r}$ is the distance. These things have earlier been studied and published by Barrera and Thelin ( 4 ) in detail for the rotation velocity studies in galaxies, where a new formula has been presented. A summary of these studies is presented here.

By using a polar coordinate system with $\mathrm{d} \theta=0$ the following expression from equation (8) is obtained :

$$
d s^{2}=-\gamma^{-1} d r^{2}-r^{2} d \theta^{2}+\gamma d t^{2}
$$

where $\gamma=(\mathbf{1}-\mathbf{2 M} / \mathbf{r})$, which is the Schwarzschild term Eddington (11)(p.82-85)

From this formula it is possible to obtain an expression for the angular rotation speed of a galaxy :

$$
\mathrm{d \theta}=(1 / \mathrm{r})\left((1-2 \mathrm{M} / \mathrm{r}) \mathrm{dt}^{2}-(1-2 \mathrm{M} / \mathrm{r})^{-1} \mathrm{dr}^{2}\right)^{1 / 2}
$$

If we suggest the space-time to be constant for a galaxy the $\mathrm{dr}=0$ which gives

$\mathrm{dr}^{2}=0$. This means that equation (10) can be rewritten as :

$$
d \theta=(1 / r)\left((1-2 M / r) d t^{2}\right)^{1 / 2}
$$

which gives a simpler equation of the angular rotation speed of galaxies :

$$
\mathrm{d} \theta / \mathrm{dt}=(1 / \mathrm{r})(1-2 \mathrm{M} / \mathrm{r})^{1 / 2}
$$

By introduction of a scale factor $\mathrm{k}$ according to will lead to the following appearance : $\quad \mathrm{d} \theta / \mathrm{dt}=(\mathrm{k} / \mathrm{r})(\mathbf{1}-\mathbf{2 M} / \mathrm{r})^{1 / 2}$

The formula of the rotation speed $(\mathrm{km} / \mathrm{s})$ is obtained from the expression 


$$
\mathbf{v}=\mathbf{r}(\mathrm{d} \theta / \mathrm{dt})
$$

This means that the rotation speed in $\mathrm{km} / \mathrm{s}$ will be the following expression :

$$
v=k(1-2 M / r)^{1 / 2}
$$

\section{The new universal formula}

Exact solution of complete relativity for polar coordinates Minkowsky/Schwartschild metric gives for non geodetic lines(Inverse lines)

$d s^{2}=-\gamma^{-1} d r^{2}-r^{2} d \theta^{2}+\gamma d t^{2}$

According to the gravitation tensor of Einstein $\mathbf{G}_{\boldsymbol{\mu}}=\mathbf{0}$ which is symmetric and gives possibility to the interchange between the $t$ and $s$ variables.

This interchange between the $\mathrm{s}$ and $\mathrm{t}$ variables gives the rotation velocity in arc-length parameters :

$\mathrm{dt}^{2}=-\gamma^{-1} \mathrm{dr}^{2}-\mathbf{r}^{2} \mathrm{~d} \boldsymbol{\theta}^{2}+\gamma d \mathrm{~s}^{2}$

Rearranging the order between the parameters involved gives :

$r^{2} d \theta^{2}=-\gamma^{-1} d r^{2}+\gamma d s^{2}-d t^{2}$

By dividing with ds we obtain :

$\mathbf{r}^{2}(\mathrm{d \theta} / \mathrm{ds})^{2}=-\gamma^{-1}(\mathrm{dr} / \mathrm{ds})^{2}+\gamma-(\mathrm{dt} / \mathrm{ds})^{2}$

From this we obtain the relativistic rotation speed $\mathbf{v}_{2}=\mathbf{r}(\mathbf{d \theta} / \mathbf{d s})=\left(-\gamma^{-1}(\mathrm{dr} / \mathrm{ds})^{2}+\gamma-(\mathrm{dt} / \mathrm{ds})^{2}\right)^{1 / 2}$ (20)

By assuming that we have circular orbit follows $(\mathbf{d r} / \mathbf{d s})=\mathbf{0}$ which gives :

$v_{2}=r(d \theta / d s)=\left(\gamma-(d t / d s)^{2}\right)^{1 / 2}=\left(1-2 M / r-(d t / d s)^{2}\right)^{1 / 2}$

(relativistic part)

The rotation velocity of a solar system with relatively "small" mass, where the planets are following geodetic lines, the "Keplerian" velocity in Eddington (11) is given

$\mathbf{v}_{1}=(\mathrm{M} / \mathbf{r})^{1 / 2}$

This occurs for masses less than $10^{35}-10^{39} \mathrm{~kg}$. We now want to merge these formulas into one single formula, interpolating between them and thus creating a so called "balancing scale":

$\mathbf{v}_{\text {tot }}=w_{1} v_{1}+w_{2} v_{2}$

where $\mathrm{w}_{1}$ and $\mathrm{w}_{2}$ are balancing scaling factors between the different rotation velocities $\mathrm{v}_{1}$ and $\mathrm{v}_{2}$.

First we have to transform the equation

$v_{2}=\left(1-2 M / r-(d t / d s)^{2}\right)^{1 / 2}$

into

$\left.\mathbf{v}_{2}=\left(\mathbf{v}_{\max }^{2}-M / r\right)\right)^{1 / 2}$

Since $v_{1}=(\mathbf{M G} / \mathbf{r})^{1 / 2} \quad(\mathrm{SI}-$ units $)$ means that :

$=c^{2}-2 M G / r-(d t / d s)^{2}=v_{\max }^{2}-M G / r=v_{\max }^{2}-v^{2}$

This means that $\mathbf{c}^{2}-\left(\mathbf{v}_{\max }^{2}+\mathbf{v}_{1}^{2}\right)=(\mathbf{d t} / \mathbf{d s})^{2}$

or by taking the square root:

dt $/$ ds $=\left(c^{2}-\left(v_{\max }^{2}+v^{2}\right)\right)^{1 / 2}$

so the balance is kept by the relation

$\mathrm{v}_{1}^{2}+\mathrm{v}_{2}^{2}=\mathrm{v}_{\max }^{2}$

For some constant $\mathrm{p}$ we have : $\mathbf{p}^{2}\left(\mathbf{v}_{\max }{ }^{2}-\mathbf{v}_{1}{ }^{2}\right)=\left(\mathbf{c}^{2}-\mathbf{p}^{2} \mathbf{v}^{2}\right)$

where $v_{2}$ is a multiple of the famous formula for the proper velocity in the theory of special relativity

$\mathbf{v}$ (proper) $=\left(1-\mathbf{v}^{2} / \mathbf{c}^{2}\right)^{1 / 2}$

Now it exist some 'multiple' of $\left(\mathbf{v}_{\max }{ }^{2}-\mathbf{v}_{1}{ }^{2}\right)$ equals $\left(\mathbf{c}^{2}-\mathbf{v}^{2}\right)$

for some variable $\mathrm{v}$.

Now we weight the solution such that we obtain the new universal rotation formula:

$v_{\text {tot }}=w_{1}(M G / r)^{1 / 2}+w_{2}\left(v_{\max }^{2}-M G / r\right)^{1 / 2}$

\section{Results and figures by the new rotation universal formula}

In this section "experimental" results from computer simulations made are presented.

All the computer simulation graphs are obtained by the new universal velocity distribution formula in equation ( 33 ).

The results from the atomic world are presented in Figs 1 and 2 in 2 and 3 dimensions respectively. 


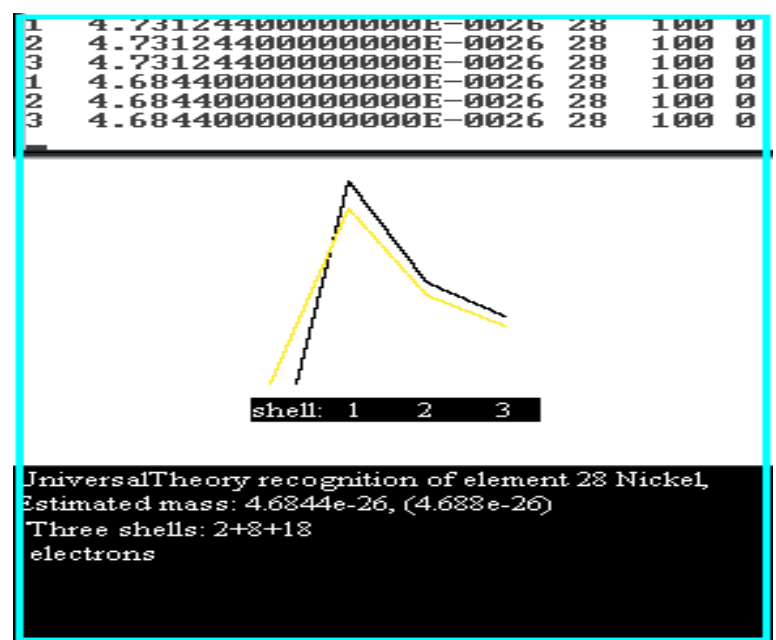

Fig 1 The rotation velocity $v$ versus distance $r$ and principal quantum number $n$ for electrons in the atoms versus distance $r$ to the nucleus of the atom.

The $\mathrm{v} \alpha(1 / \mathrm{r})^{1 / 2}$ dependence and the $\mathrm{v} \alpha(1 / \mathrm{n})$ dependence are clearly seen.

(Computer simulations)

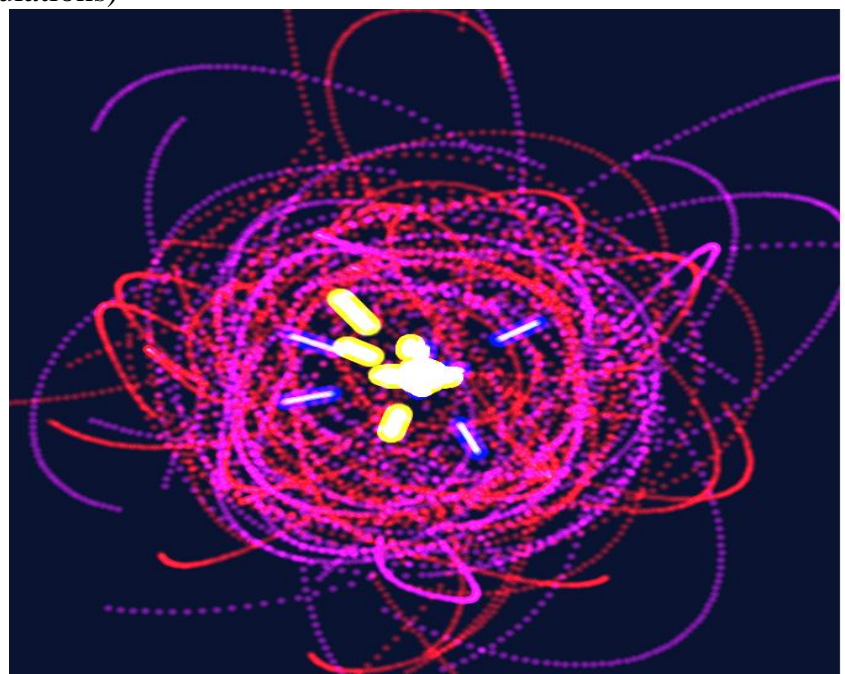

Fig 2 The rotation velocity versus distance for electrons in the atom in 3 dimensions

The shell structure is clearly seen. (Computer simulations)

For the planets the following rotation velocity distributions are obtained.

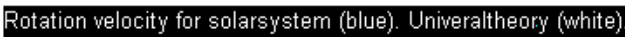

Estimated mass of solarsystem by Universal theory $=1.998 \mathrm{e}+30 \mathrm{~kg},(2 \mathrm{e}+30 \mathrm{~kg})$

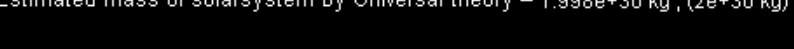

IAE

$30 \mathrm{AE}$
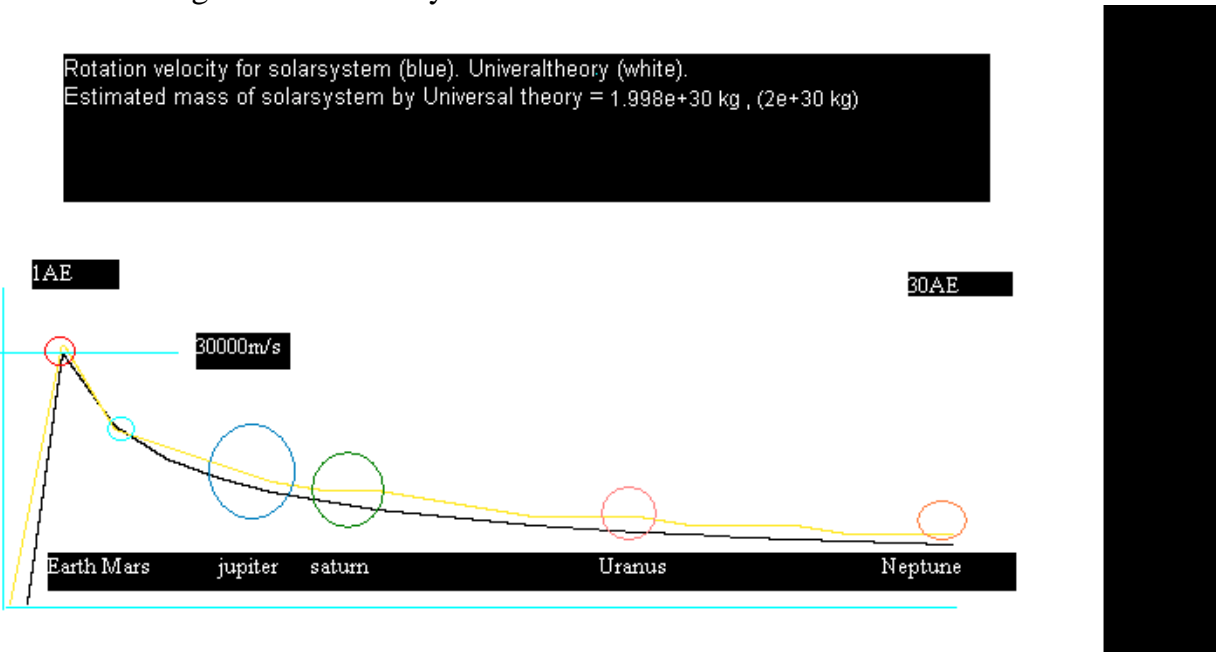

Fig 3 Velocity distributions versus distance of the largest planets (Jupiter, Saturn, Uranus, and Neptune ) (Computer simulations) 


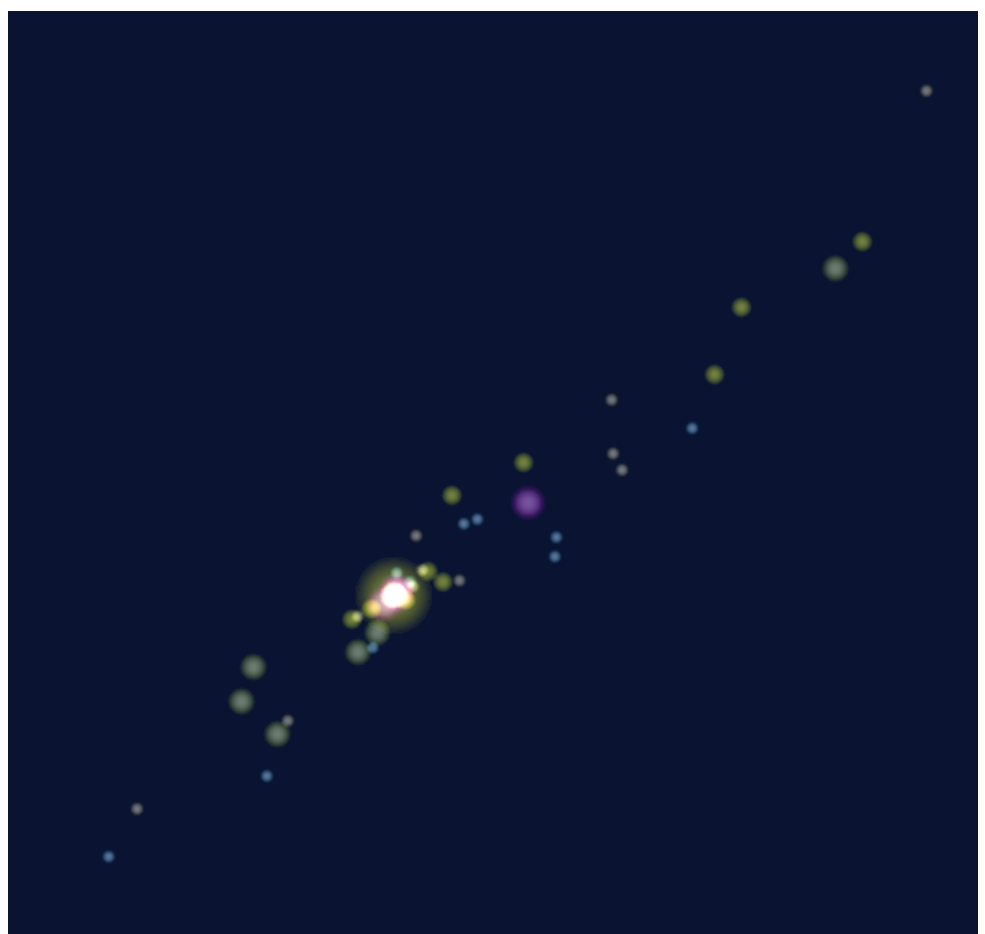

Fig 4 Velocity distributions of some planets in 3 dimensions.

The planet orbits in one plane are clearly seen. (Computer simulations)

For the galaxies the following rotation velocity distributions are obtained.
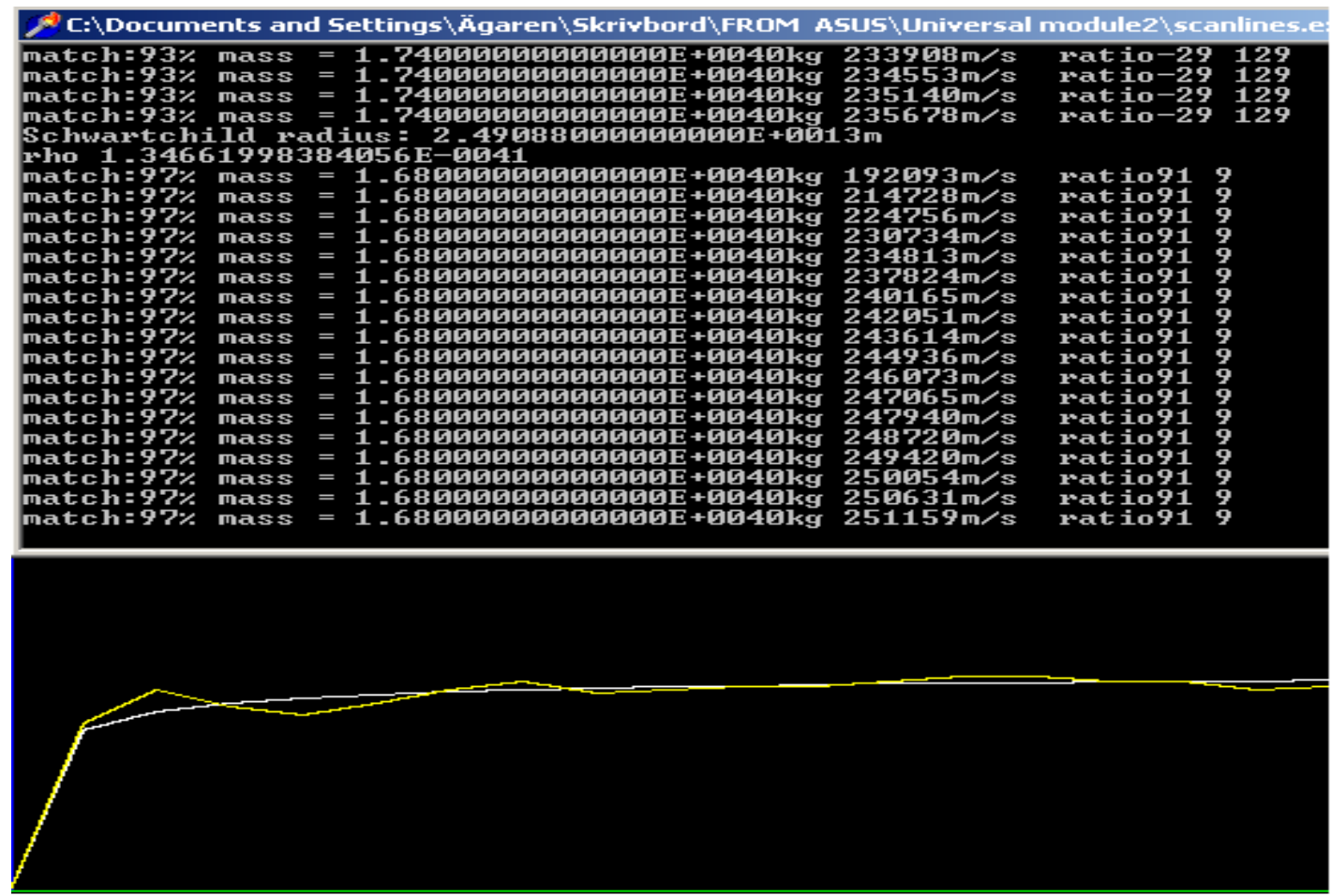

\section{Galaxy NGC801}

Fig 5 Velocity distributions versus distance of the galaxies NGC( 801 ) These graphs do follow the observational velocity distributions.

(Computer simulations) Match 97\% Equation 33 has been followed 

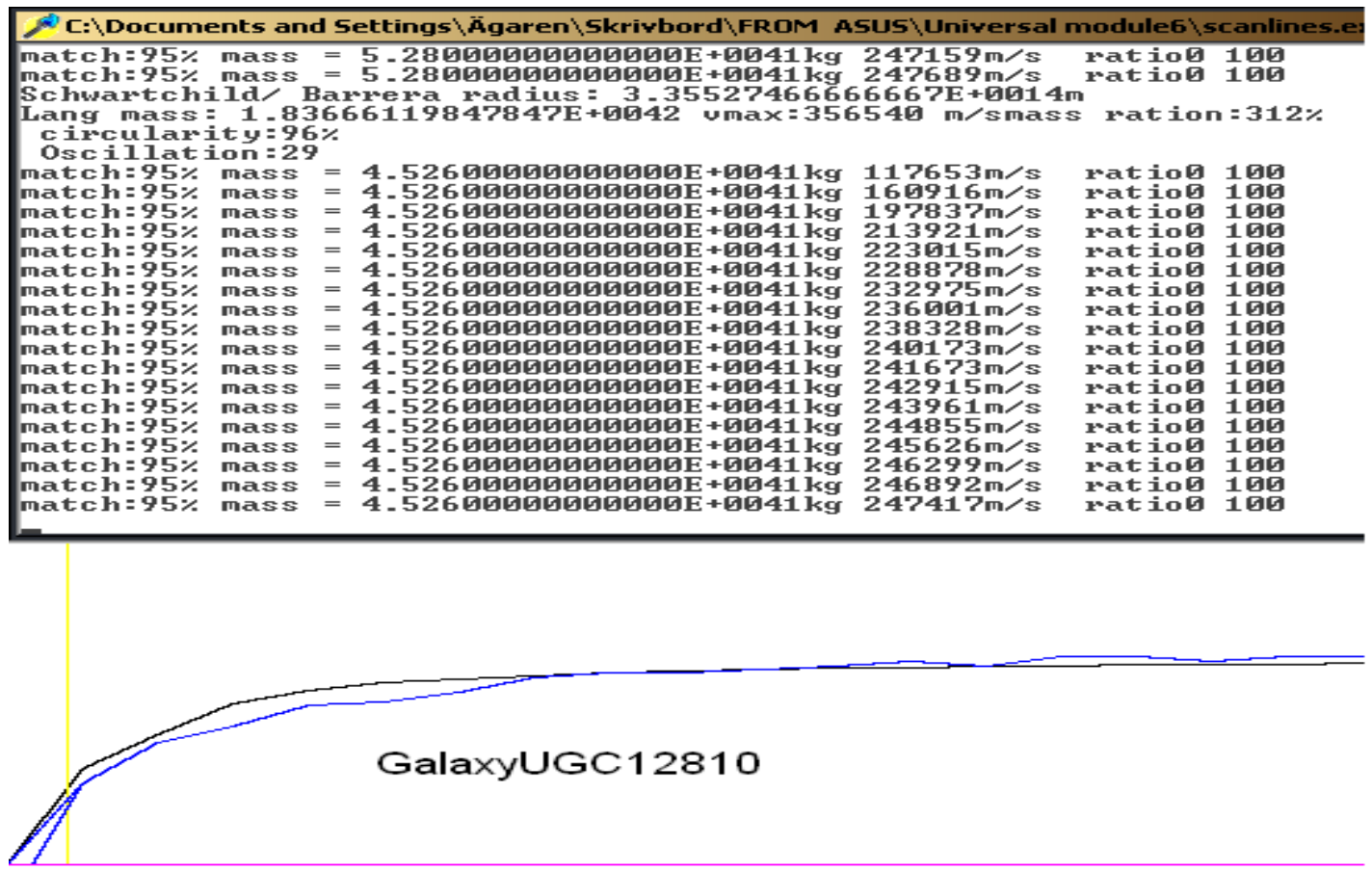

Fig 6 Velocity distributions versus distance of the galaxies UGC(12 810)

These graphs do follow the observational velocity distributions.

(Computer simulations) Equation 33 has been followed

Match $95 \%$

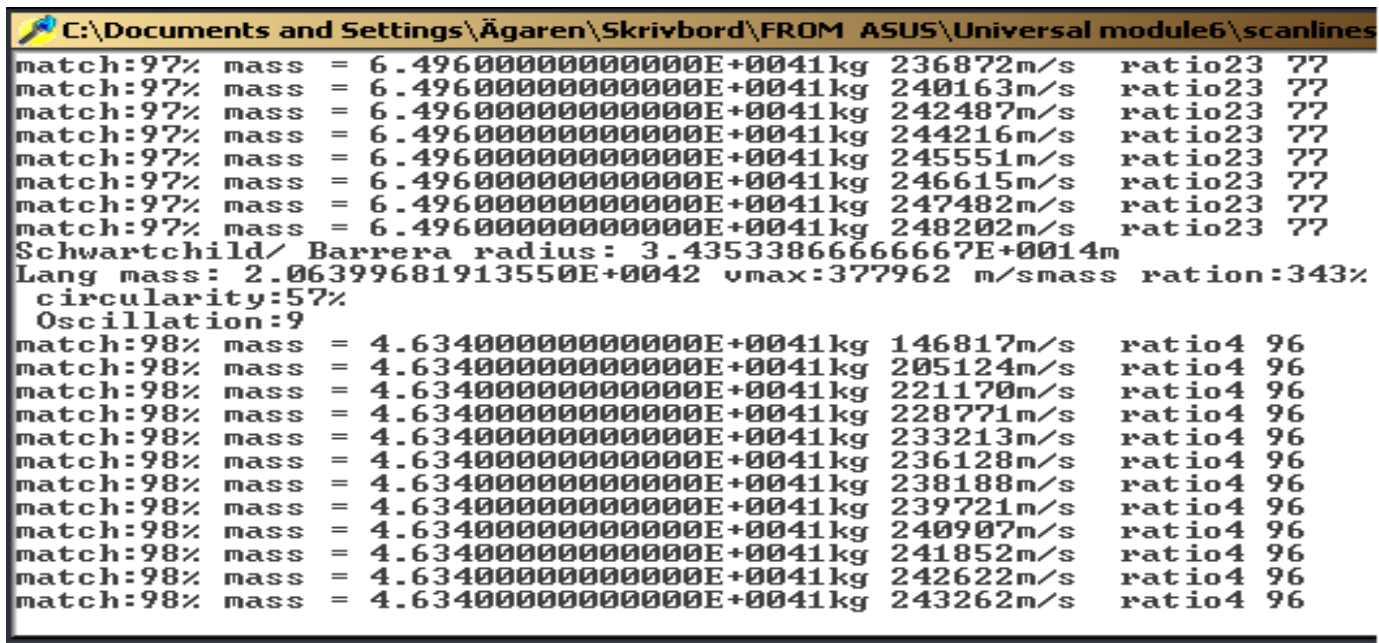

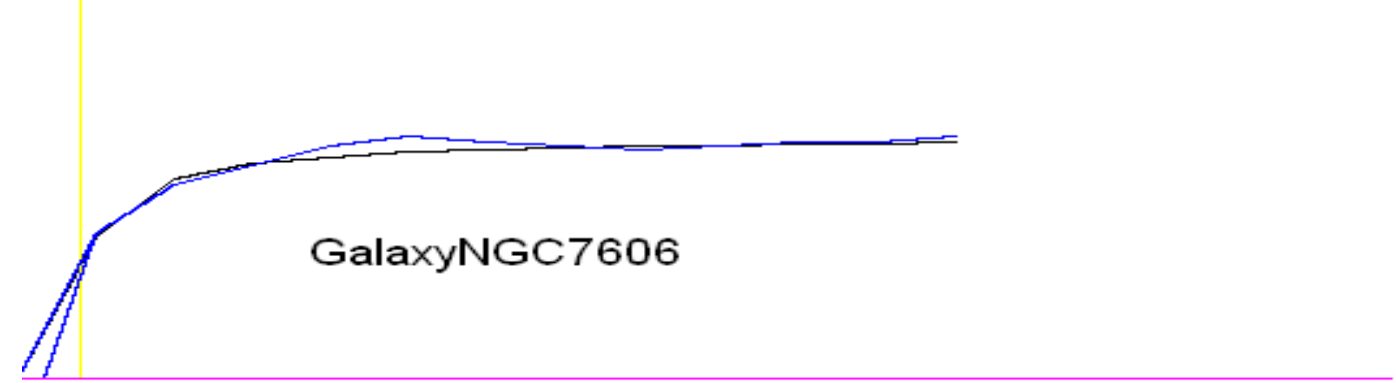

Fig 7 Velocity distributions versus distance of the galaxies NGC7606 These graphs do follow the observational velocity distributions.

(Computer simulations) Equation 33 has been followed

Match 98\% 


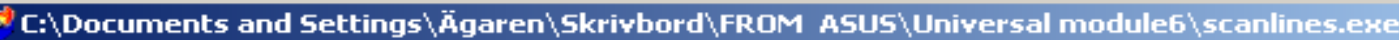

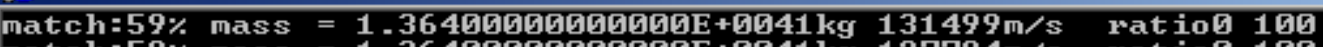

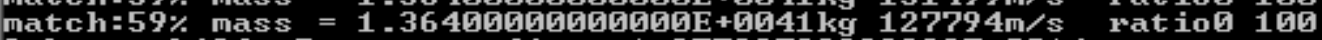
Schwartchild/ Barrera radius: 1 - $37739733333333 \mathrm{E}+0 \mathrm{~g} 14 \mathrm{~m}$

Lang mass : 1 -89391579702788E+0042 umax=362055 m/smass ration = $784 \%$ circularity: $67 \%$

Oscillation 47

match:59\% mass $=1.85800000000000 \mathrm{E}+0041 \mathrm{~kg} 523788 \mathrm{~m} / \mathrm{s}$ match=59\% mass $=1.85800000000000 \mathrm{E}+0041 \mathrm{~kg} 370374 \mathrm{~m} / \mathrm{s}$

match:59\% mass $=1.8580$ GagagagagaE $+0041 \mathrm{~kg} 362409 \mathrm{~m} / \mathrm{s}$

match=59\% mass $=1.8580$ gaglaglagaE $+0041 \mathrm{~kg} 261894 \mathrm{~m} / \mathrm{s}$

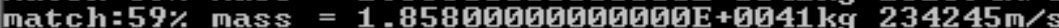

match:59\% mass $=1.8580$ Gabagagag $+0041 \mathrm{~kg} 213835 \mathrm{~m} / \mathrm{s}$

match=59\% mass $=1.85800000000000 \mathrm{E}+0041 \mathrm{~kg} 197973 \mathrm{~m} / \mathrm{s}$

match=59\% mass = 1.8580 agGagagagaE $+0041 \mathrm{~kg} 185187 \mathrm{~m} / \mathrm{s}$

match:59\% mass = 1.8580 G

match:59\% mass = 1.858000 Jaglagage $+0041 \mathrm{~kg} 165636 \mathrm{~m} / \mathrm{s}$

match=59\% mass $=1.8580$ abagabagaE $+0041 \mathrm{~kg} 157928 \mathrm{~m} / \mathrm{s}$

match=59\% mass $=1.85800000000000 \mathrm{E}+0041 \mathrm{~kg} 151204 \mathrm{~m} / \mathrm{s}$

match=59\% mass $=1.85800000000000 \mathrm{E}+0041 \mathrm{~kg} 145273 \mathrm{~m} / \mathrm{s}$

match:59\% mass $=1.8580$ gagagagagaE $+0041 \mathrm{~kg} 139988 \mathrm{~m} / \mathrm{s}$

match=59\% mass $=1.85800000000000 \mathrm{E}+0041 \mathrm{~kg} 135241 \mathrm{~m} / \mathrm{s}$

match:59\% mass $=1.8580$ abagagaga $+0041 \mathrm{~kg} 130947 \mathrm{~m} / \mathrm{s}$

match:59\% mass $=1.85800000000000 \mathrm{E}+0041 \mathrm{~kg} 127037 \mathrm{~m} / \mathrm{s}$

match:59\% mass $=1.85800000000000 \mathrm{E}+0041 \mathrm{~kg} 123458 \mathrm{~m} / \mathrm{s}$

ratiog 190

ratiog 1 ag

ratiog 109

ratiog 100

ratiog 109

ratiog 190

ratiog 1 Ga

ratiog 100

ratiog 190

ratiog 100

ratiog 109

ratiog 190

ratiog 1 Ga

ratiog 190

ratiog 100

ratiog 109

ratiog 1 Ga

ratiog 100

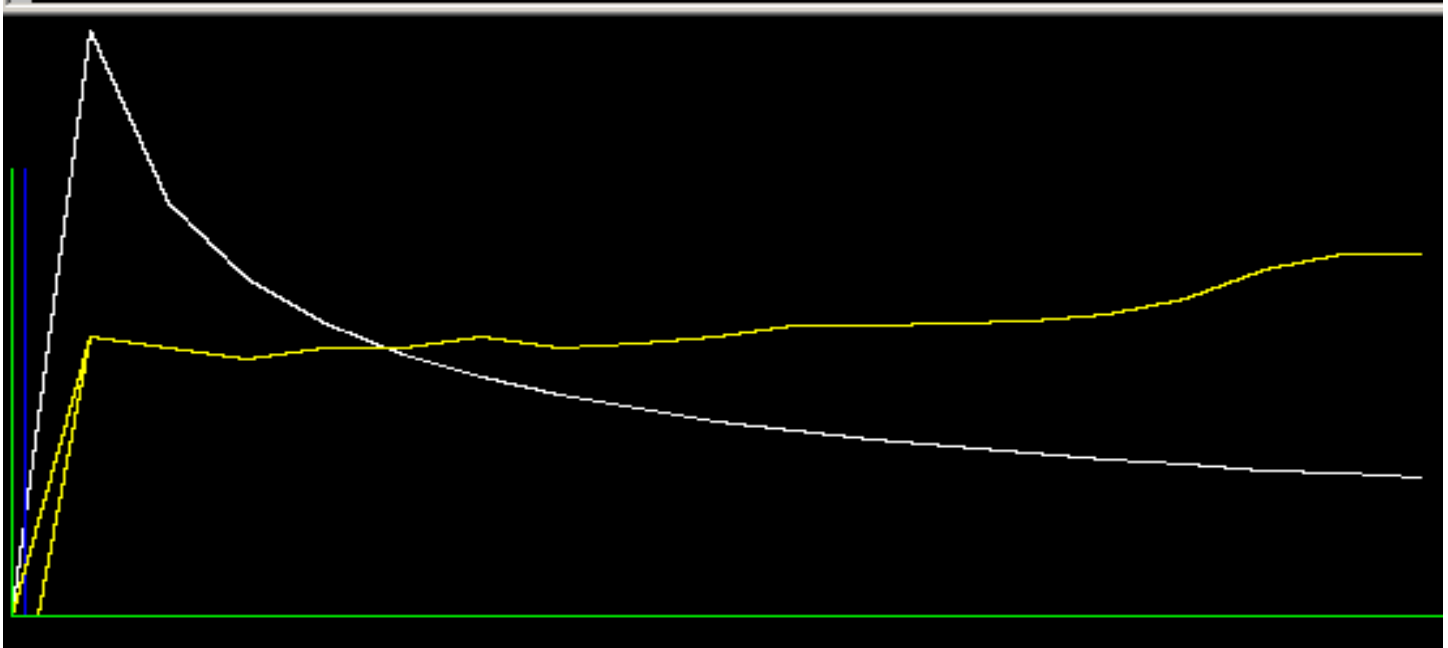

Fig 8 Velocity distributions versus distance of the galaxies NGC Milky Way

These graphs do not follow the observational velocity distributions.

Equation 2 has been followed. Theoretical=white curve

(Computer simulations) Match 59\%

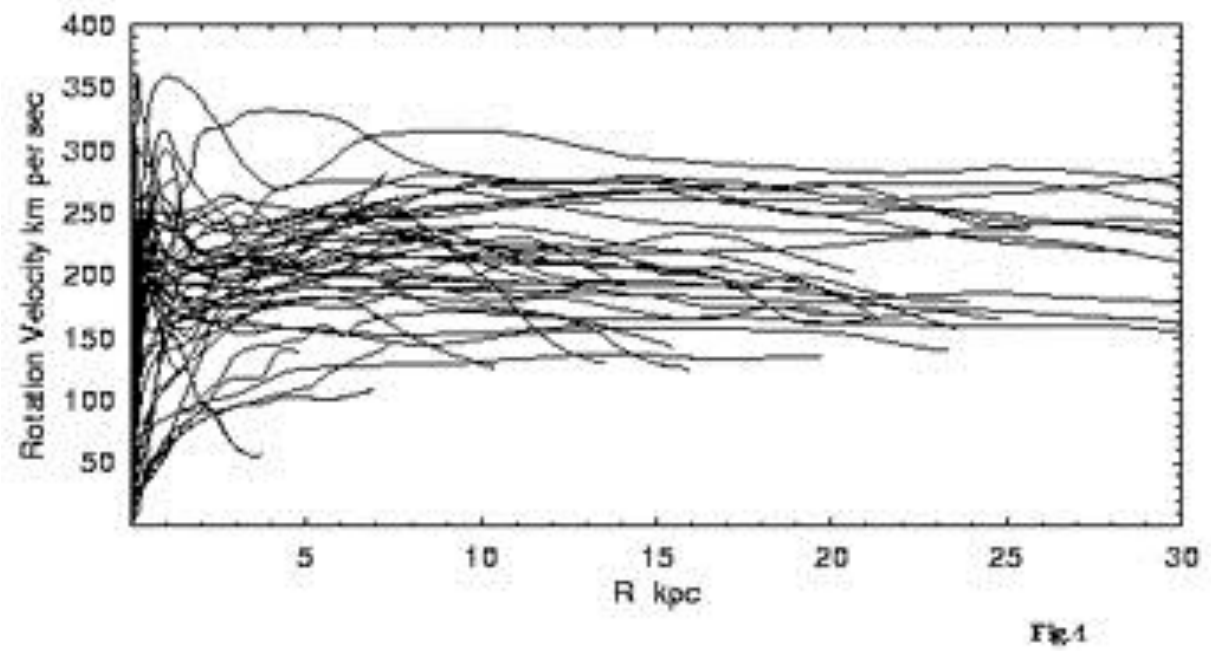

Fig 9 Observational results of velocity distributions versus distance of many galaxies.

Reproduction from Sofue and Rubin.( 7 ) 


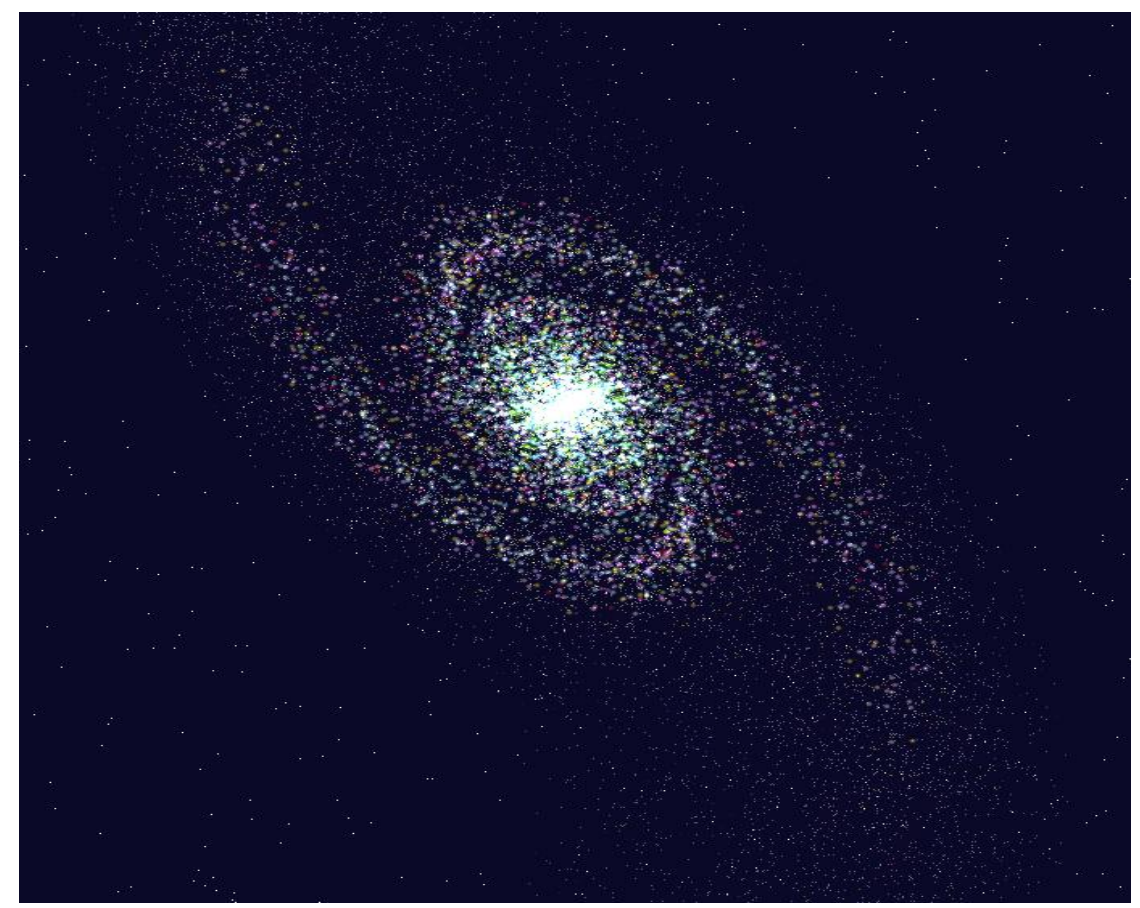

Fig 10 Computer simulations of randomly distributed 30000 "stars" distributed like an ellipsoid in a galaxy. The appearance is very similar to observations of galaxies.

Reproduction from Barrera and Thelin ( 4 )

Table 1

Matching in the observational and theoretical velocity versus distance graphs on galaxies

$\begin{array}{lccccc}\text { Galaxnr } & \text { match } \% & \text { radius } & \mathrm{v}_{\max } & \text { Estim.Mass(eq 33) } & \text { Appr.Mass (3) } \\ \text { NGC3200 } & 93 & 1.8010^{5} \mathrm{ly} & 2.8010^{5} \mathrm{~m} / \mathrm{s} & 4.2910^{41} \mathrm{~kg} & 1.2710^{42} \mathrm{~kg} \\ \text { NGC801 } & 97 & 1.8010^{5} & & 3.6510^{41} & \text { " } \\ \text { UGC2885 } & 90 & 2.2010^{5} & 3.2510^{5} & 6.9910^{41} & 2.1010^{42} \\ \text { Milky Way } & 92 & 0.8010^{5} & 3.0010^{5} & 4.0210^{41} & 6.5010^{41} \\ \text { NGC7606 } & 98 & 1.2010^{5} & 2.5010^{5} & 4.6310^{41} & 6.2010^{41} \\ \text { UGC12810 } & 95 & 1.8010^{5} & 2.0010^{5} & 5.2410^{41} & 6.5010^{41} \\ \text { NGC1417 } & 92 & 1.0010^{5} & 3.2510^{5} & 6.8810^{40} & 9.5410^{41}\end{array}$

It is also possible to calculate the Radius, $\mathrm{V}_{\max }$, Estimated Mass of the galaxies studied. By using equation 33 very good match percentage is achieved. The approximated mass values are following equation 2 from Combes(3) and have higher values.

\section{Discussion}

For atoms the rotation velocity of the electrons versus distance to the nucleus expression were done by Niels Bohr by using Coulombs law and the circular centripetal force of an electron around an atom. These calculations were done to achieve the shell structure of the atoms and are established fact today and are seen in equation 3. This formula follows the Keplerian relationship $\mathrm{v} \alpha\left(1 / \mathrm{r}^{1 / 2}\right)$ between rotation velocity and distance to the nucleus of the atom. By using equation 33 in this paper together with computer simulations we have achieved similar atomic structure and appearance as the usual picture of an atom (Figs (1 and 2) in 2 and 3 dimensions. Fig 1 also illustrates $\vee \propto 1 / \mathrm{n}-$ dependence of the rotation speed versus the principal quantum number, also in accordance with the literature.

An established fact is that planets follow the 3:rd law of Kepler as can be seen in equation(7) for circular motion, where $\mathrm{V}$ is the planet rotation speed around the sun and $\mathrm{m}$ is the planet mass and $\mathrm{M}$ is the sun mass. By using equation 33 and computer simulations this is illustrated in Figs 3 and 4 in 2 and 3 dimensions. In Fig 3 the big planets Jupiter, Saturn, Uranus and Neptune illustrate a decreasing rotation speed around the sun versus distance to the sun. Fig 4 illustrates a planetary system, which has a rather flat structure in accordance with our planetary system in 3 dimensions. 
We can se from sections 2 and 3 from Barrera and Thelin ( 4 ) that the velocity formula between velocity and distance to the center of the galaxy has a $\sqrt{x}$ - structure. These facts are based on results presented in Combes ( 3 ) and Lang (10) and are not based on Kepler`s 3:rd law directly. These relationships are observed by the astronomers where the velocity reaches a constant speed between 5 and $10 \mathrm{kpc}$ from the centre of the galaxy. A similar structure is also obtained by using the theory of relativity and the Schwarzschild metric in equation 19 of Barrera and Thelin ( 4 ) and from the new expanded formula (33) of this paper and is seen in Figs (5-7 ) where a steep rising of the velocity (angular and circular in $\mathrm{km} / \mathrm{s}$ ) at low distances is observed. After that rising, a plateau is reached, which will be dominating up to large distances. Our model is also in accordance with the density distribution in the galaxy which shows a decreasing profile to distance, which is in accordance with earlier observations. We have also used equation 33 and computer simulations of a galaxy from above giving a realistic structure (Fig 10). Very good observational graphs of velocity-distance curves are seen in Fig 9 and have the same profile as our Figures (5-7).

This means that equation (33) has the capacity to illustrate completely different objects (atoms to galaxies) in nature in a realistic way.

In Table 1 it is also possible to calculate the Radius, $\mathrm{V}_{\max }$, Estimated Mass of the galaxies studied. By using equation 33 very good match percentage is achieved. The approximated mass values are following equation 2 from Combes(3) and have higher values. If one uses equation 2 instead in the matching procedure bad correlation is achieved, which is seen in Fig 8. For a number of galaxies measured the mean value of matching with equation 33 is $94 \%$ and with equation 2 is 55\%. Computer simulations were also performed to establish and verify the rotation velocity and mass in the galaxy, suggested in this paper and giving a strong support of the new formulas and the use of the theory of relativity.

The observations from Freeman (1) and Rubin (2) are also observed by many astronomers and have been a controversial discovery, because it contradicts Kepler`3:rd law, which will follow a $(1 / \sqrt{r})$ dependence and is not observed in any galaxies. Therefore, some astronomers claim that there must be a large amount of dark matter in the galaxy, which is the cause of this discrepancy and also holds the galaxies together at those high rotation speeds.

Similar $\sqrt{x}$ - structures of the velocity curves as our curves have also been obtained in the so called Mond- project, where a modification of the Newton`s law is applied by Sanders (12).

It can be noted that our solution in equation 33 is a solution of the Chebyshev differential equation found in common mathematical handbooks.

We want to thank :

Acknowledgement :

Dr. Gunnar Berg at the Mathematical Institution at Uppsala University,

Prof. Christer Kiselman at the Mathematical Institution at Uppsala University,

Dr. Anders Hast at Creative Media Lab at University of Gävle.

Prof. Ewert Bengtsson at Centre for Image Analysis at Uppsala University

Dr.Staffan Yngve at the Physical Institution at Uppsala University

Computer Expert Michael Nordstedt Computer Program and System support, BMC Uppsala University

for valuable discussions and critical examinations of the material in this paper.

Freeman, K. C. ,(1970), Ap. J.,160, 811

\section{References :}

Rubin, V. C., Ford, W. K., (1970), Ap, J. 150, 379

Combes, F., Boisse`,P., Mazure ,A. and Blanchard, A., Galaxies and Cosmology,(2001)

Barrera,T.,Thelin,B.,IOSR Journal of Applied Physics (IOSR-JAP) Vol 3 (4) p.44 (2013)

Schwarzschild, K., (1916),Sitz. Acad. Wiss., Physik-Math., Kl. 1, 189

Einstein, A., (1916), Ann. Physik, 49, 769

Sofue, Y., Rubin, V.C., Annu.Rev.Astronom.Astrophys.2001,Vol 39, p. 137-174

Gasiorowicz, S., Quantum Physics 3:rd Ed. (2003)

Lang, K.R., (1974) Astrophysical Formula

Lang, K.R.,(1998), Astrophysical Formula, Vol. 2

Eddington, A.S.(1923), Mathematical Theory of Relativity

Sanders, R., H., (2002) Annu. Rev. Astron. Astrophys. Vol 40,p.263-317 